\title{
Abordaje anterior directo modificado en cirugía de cadera con técnica de mínima invasión y uso de mesa especial: descripción de la técnica
}

\section{Modified direct anterior approach in hip surgery with minimally invasive surgery technique and the use of a special table: technique description}

\author{
Luis Justino Fernández Palomo, ${ }^{*}$ Daniel Diego Ball, ${ }^{\star}$ Ramón González Pola*
}

Citar como: Fernández PLJ, Diego BD, González PR. Abordaje anterior directo modificado en cirugía de cadera con técnica de mínima invasión y uso de mesa especial: descripción de la técnica. An Med ABC. 2021; 66 (2): 104-109. https://dx.doi.org/10.35366/100478

\section{RESUMEN}

El abordaje anterior directo modificado en cirugía de reemplazo total de cadera se caracteriza por la preservación de la integridad de los grupos musculares y sus inserciones, con lo cual se favorece una rápida recuperación y menor número de complicaciones. Este abordaje ha sido popularizado en las últimas dos décadas y cada vez empleado por más cirujanos en el mundo. Describimos la técnica de mínima invasión modificada, en la cual se ha sistematizado el uso de una mesa especial y en la que se han modificado algunos pasos como la realización de capsulotomía y no capsulectomía, así mismo la osteotomía del cuello femoral se realiza in situ. El uso de la mesa Hana ${ }^{\circledR}$ o Medacta ${ }^{\circledR}$, dependiendo el sistema seleccionado, permite control de tracción y rotación de la extremidad durante el procedimiento. Se describe el uso reducido de separadores especiales, así como un retractor Charnley modificado para la exposición eficiente. El abordaje anterior presenta ventajas significativas como un menor tiempo de recuperación, menor nivel de dolor, mejoría en la satisfacción del paciente y una adecuada colocación y posicionamiento de los implantes. Esta revisión presenta varios aspectos del abordaje anterior modificado para artroplastia de cadera utilizado por los autores principales durante los últimos tres años.

Palabras clave: Abordaje anterior, mesa especial Hana, Medacta, artroplastia de cadera.

Nivel de evidencia: III

\section{ABSTRACT}

The anterior approach in hip replacement surgery preserves the integrity of muscle groups and their insertions, facilitating a more rapid recovery process and minimization of postoperative complications. This approach has been popularized during the last two decades and recently performed by more surgeons around the world. We present the minimally invasive technique with a special table used and in which some steps of the original anterior approach have been modified as a capsulotomy instead of a capsulectomy, and the femoral neck osteotomy done in situ. The use of the Hana ${ }^{\circledR}$ or the Medacta ${ }^{\circledR}$ table for leg positioning permits an adecuate traction and rotation control during the procedure. We describe the reduced need of special retractors and the use of a modified Charnley retractor for exposure. The advantages of the anterior approach are: less recovery time, lower postoperative pain, better patient satisfaction and an correct prosthetic component position. This review describes several aspects regarding the modified anterior approach for hip replacement used by the senior authors for the last three years.

Keywords: Anterior approach, special table Hana, Medacta, hip replacement.

Level of evidence: III
* Ortopedia y Traumatología, adscrito al Centro de Ortopedia y Traumatología del Centro Médico ABC.

Recibido: 08/04/2021. Aceptado: 01/06/2021.
Correspondencia:

Luis Justino Fernández Palomo

E-mail: ljfernandez@abchospital.com 


\section{INTRODUCCIÓN}

Se han descrito diversos abordajes quirúrgicos para artroplastia total de cadera, como el posterior (Moore o Sureño), lateral (Hardinge), anterolateral (Hardinge), y el abordaje anterior directo (SmithPeterson); este último fue descrito originalmente por Carl Hueter en 1881 y posteriormente popularizado por Smith-Peterson después de publicar su primera descripción en $1917,{ }^{1}$ utilizando el intervalo muscular entre el tensor fascia lata y el músculo sartorio. Light y Keggi publicaron su experiencia utilizando este enfoque para artroplastia de cadera en 1980 y Judet describió el procedimiento con el uso de una mesa de fracturas en $1985 .^{2}$ La tendencia por parte de los cirujanos de cadera en poder ofrecer y realizar una artroplastia de cadera con técnicas menos invasivas ha sido el factor determinante para el renovado interés en este abordaje.

Durante los últimos 25 años se han descrito variaciones de la técnica para una exposición segura y exitosa de la cadera durante la artroplastia, ${ }^{3}$ siendo utilizada últimamente también de forma rutinaria para cirugías de revisión y hemiartroplastia en fracturas. ${ }^{4}$

Joel Matta en Los Ángeles, California, Estados Unidos $^{5,6}$ y Frederic Laude en Paris, Francia, ${ }^{7}$ ambos discípulos del profesor Emile Letournel, han realizado importantes aportaciones en el uso de mesas especiales que permiten un cómodo y favorable posicionamiento de la extremidad afectada para la manipulación y colocación de los implantes. La técnica AMIS descrita por Laude remarca una diferencia importante en la disminución del número de separadores empleados y en la secuencia del acceso intermuscular, el cual se realiza entre el músculo tensor de la fascia lata y el recto anterior, a diferencia de la técnica convencional en la que se aborda entre el recto anterior y el sartorio. El uso de mesa o posicionador especial como son la mesa Hana ${ }^{\circledR}$ (Mizuho, EUA) o el posicionador de Medacta ${ }^{\circledR}$ (Medacta International, Suiza) facilitan el procedimiento, ya que disminuyen el número de manos necesarias, mantienen constante la posición del fémur y permiten la manipulación de la extremidad para las maniobras de luxación y reducción de la cadera.

\section{TÉCNICA QUIRÚRGICA}

$\mathrm{El}$ abordaje anterior se realiza por un intervalo intermuscular e internervioso, reduciendo potencialmente el riesgo de daño a estructuras periarticulares, incluidos músculos, tendones, vasos femorales y nervios.

\section{Posicionamiento del paciente}

El paciente se coloca en decúbito supino mesa ortopédica especial Hana o de Medacta dependiendo el sistema seleccionado para la artroplastia. Con un soporte perineal (Figuras 1 y 2) que debe tener unos $10 \mathrm{~cm}$ de diámetro y, siempre que sea posible, estar recubierto con un gel para proteger la zona. La mesa Hana ${ }^{\circledR}$ fue desarrollada por el Dr. Joel Matta inspirado en las mesas de tracción para cirugía de pelvis como la mesa de Judet, es un equipo con sistemas hidráulicos y electrónicos automatizados que permiten una movilización suave de las extremidades y facilitan la movilización por parte del personal auxiliar. La mesa Medacta ${ }^{\circledR}$ es más bien un posicionador de pierna adaptable a cualquier mesa de quirófano convencional. Ésta puede ser transportada muy fácilmente y su manejo es muy sencillo, facilitando al personal auxiliar su manipulación. El pie en el lado operatorio está asegurado por una bota especial, luego se aplica tracción a las extremidades, para obtener una posición pélvica sin basculación y horizontal. La extremidad torácica no debe interferir con el campo de visión, por lo que debe ser fijada con el codo en flexión sobre el tórax. Se verifica posición con intensificador de imágenes, asegurando la adecuada simetría de la sínfisis púbica.

Se realiza lavado y vestido como parte del proceso de preparación del campo quirúrgico. Pueden emplearse campos especiales diseñados para cada mesa en particular o un sistema de cuatro campos simples de manera habitual.

\section{Incisión}

La incisión inicia $2 \mathrm{~cm}$ distales y $3 \mathrm{~cm}$ laterales a la espina iliaca anterosuperior, y continúa oblicuamen-

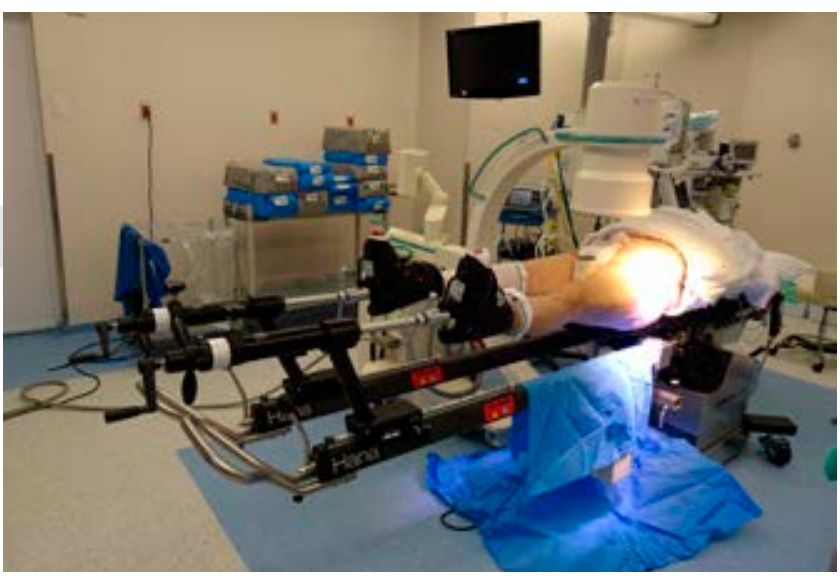

Figura 1: Mesa Hana ${ }^{\circledR}$ (Mizuho). Mesa hidráulica con control electrónico. 


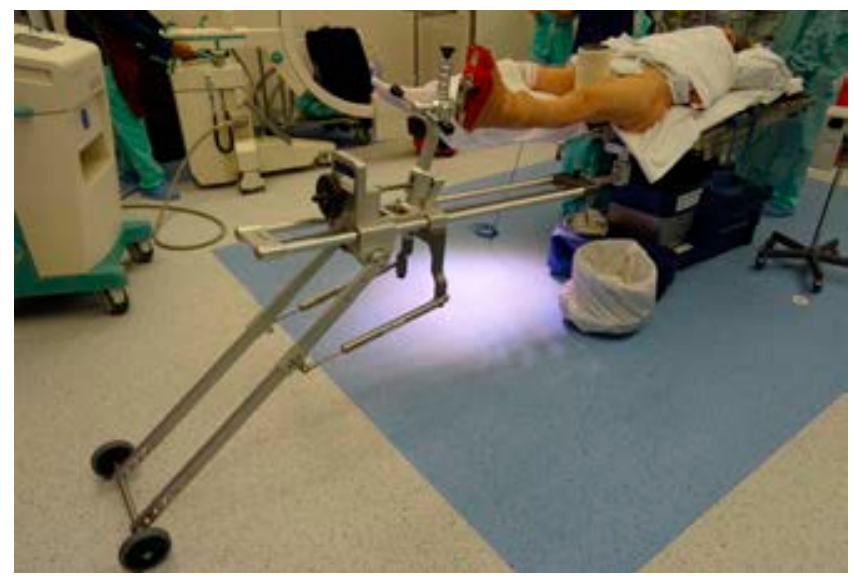

Figura 2: Mesa Medacta ${ }^{\circledR}$, posicionador de operación manual adaptable a una mesa convencional.

te entre 8 y $10 \mathrm{~cm}$ hacia distal y ligeramente hacia lateral, sobre la mitad de la masa muscular palpable del tensor de la fascia lata (Figura 3).

Después de la hemostasia del tejido subcutáneo, la vaina del tensor de la fascia lata se identifica. Es incidida longitudinalmente en una ligera inclinación hacia distal y lateral, evitando disecar hacia medial para no dañar el nervio femorocutáneo lateral. ${ }^{8}$

Se identifica el espacio intermuscular profundo, colocando retractores Beckmann especiales en el sitio. La división revela el espacio entre la aponeurosis superficial del recto femoral y el vasto lateral. Esta aponeurosis se incide longitudinalmente, el vientre del músculo se retrae medialmente para exponer la aponeurosis profunda para identificar la arteria y venas circunflejas laterales después de haber pasado entre el tensor y el recto femoral, las cuales deben ser ligadas y/o cauterizadas para evitar un sangrado excesivo.

Al ligar los vasos, se identifica el área capsular delimitada por los bordes de los vientres musculares del recto anterior e iliocapsular medialmente, el vasto lateral y distalmente, y el glúteo medio, lateral y proximalmente. Se retira el tejido graso de esta zona y se expone la cápsula articular.

\section{Abordaje articular}

Se realiza la capsulotomía en V invertida, iniciando de la espina iliaca anterosuperior hacia el trocánter menor y en sentido opuesto siguiendo el reborde de la cresta intertrocantérica del fémur, colocando separadores superolateral e inferomedial al cuello.

\section{Osteotomía del cuello femoral}

Después de la capsulotomía, es posible colocar el retractor especial de Charnley en la cápsula. El brazo más largo del retractor se coloca dentro de la cápsula medial. El asistente sostiene el retractor en esa posición, luego el cirujano coloca el segundo brazo del retractor en el colgajo capsular lateral y lo fija con presión sobre el marco de Charnley. Las dos valvas del retractor son afiladas y sólo deben ser colocadas en la cápsula. Si el retractor se desliza y toca el músculo, puede dañar estructuras anatómicas adyacentes.

El nivel de corte se identifica básicamente con referencia al ángulo cervicotrocantérico desde la planificación preoperatoria. La osteotomía se hace in situ, se coloca un osteótomo de $0.5 \mathrm{~cm}$ en el vértice del corte del borde del trocánter mayor, visualizando mediante fluoroscopia el vértice contralateral con el borde proximal del separador colocado sobre el trocánter menor. Utilizando una sierra, la posición de la extremidad inferior debe comprobarse palpando la rótula. Se realiza tracción con la mesa, lo cual favorece que al completar el corte del cuello éste se separe fácilmente.

Una vez realizada la osteotomía, se rota de manera externa la extremidad con ayuda de un asistente que manipule la mesa y se introduce un cincel en la cabeza a nivel del corte del cuello y se rota hacia medial, con esto se expone el sitio de la osteotomía y se permite la extracción de la cabeza femoral con un sacacorchos. En algunos casos, se debe liberar parte de la cápsula posterior que todavía puede estar unida al cuello femoral. El plano de corte se puede comprobar palpando directamente la distancia y dirección hacia en trocánter menor.

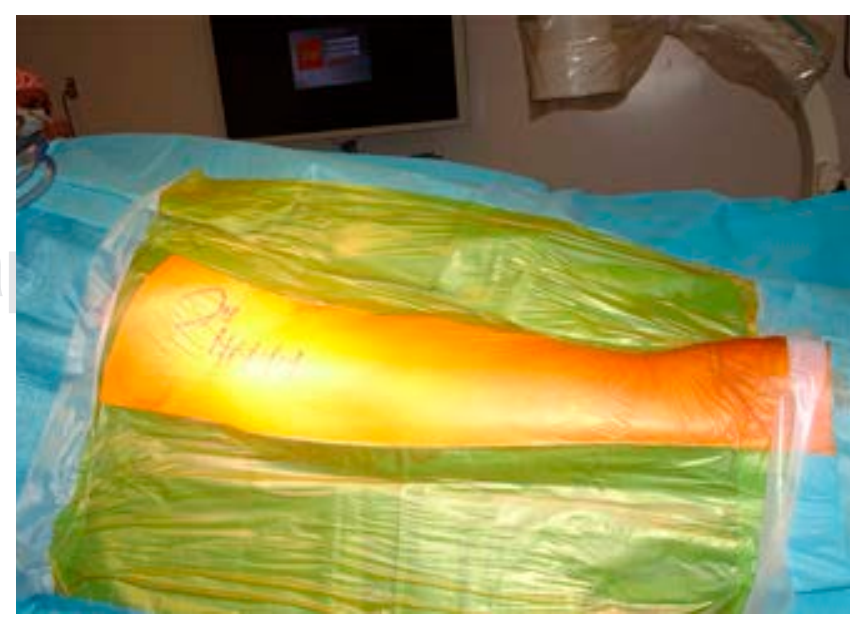

Figura 3: Incisión $2 \mathrm{~cm}$ distal a la espina iliaca anterosuperior, $3 \mathrm{~cm}$ lateral. 


\section{Preparación acetabular}

Con la extremidad a $40^{\circ}$ de rotación externa, se visualiza la cavidad acetabular. Se coloca el retractor de Charnley modificado retrayendo la cápsula anterior, con lo cual el borde acetabular es visible. Si la pared anterior del acetábulo es aún difícil de visualizar, es posible colocar un retractor de Hohmann en la porción anteroinferior del borde acetabular. Se retira el labrum y se identifica el ligamento redondo al fondo de la fosa. Se realiza electrocoagulación y se extrae. El ligamento transverso puede ser seccionado, retirado o movilizado. A continuación, se inicia el proceso de fresado; se puede utilizar un posicionador curvo con offset $\mathrm{o}$ uno recto a preferencia del cirujano y dependiendo de la complexidad del paciente para evitar lesionar la piel en el ángulo distal de la incisión o un efecto de palanca que resulta en un fresado excesivo de la pared anterior del acetábulo.

La preparación acetabular se realiza en forma convencional progresiva, se verifica la posición de las fresas previa a la colocación del implante de prueba o el definitivo mediante fluoroscopia, se debe evitar la verticalización o anteversión excesiva del implante, el cual debe posicionarse con una inclinación de 40 a 45 grados y una anteversión de 15 a 20 grados. El componente acetabular final se impacta, se realiza una prueba de extracción y se coloca el inserto acetabular. El lograr la preservación de la cápsula posterior favorece la estabilidad del implante.

\section{Preparación femoral}

Se realiza una liberación selectiva de la cápsula articular de las inserciones femorales. De las tres inserciones capsulares en el fémur, la separación de las dos inserciones anteriores (superior e inferior) facilita la rotación externa femoral. La inadecuada liberación puede resultar en una rotación externa limitada a menos de $90^{\circ}$. El ligamento iliofemoral debe liberarse de manera progresiva para poder movilizar fémur en rotación externa y extensión mientras que el desprendimiento del haz pubofemoral permite su lateralización. El haz capsular isquiofemoral no debe seccionarse de manera sistemática, éste debe ser liberado sólo en caso de que el fémur no pueda ser lateralizado y no permita el correcto posicionamiento del canal medular femoral. Si se utiliza la mesa Hana ${ }^{\circledR}$ se puede colocar un aditamento especial, el gancho femoral, que adaptado al sistema eléctrico de elevación mantiene al fémur proximal en una posición adecuada para la preparación del canal. Si no se utiliza éste sistema, puede lograrse con colocación de dos separadores de Hoffman en la región lateral del trocánter mayor y otro proximal al trocánter menor.

Se libera la tracción y se realiza extensión de la extremidad con la mesa. El plano de corte del cuello femoral es horizontal.

La preparación femoral inicia con la apertura del canal medular utilizando un instrumento iniciador, y continúa con la eliminación de hueso cortical a lo largo de la parte interna del trocánter mayor con un osteótomo de caja modificado con offset. En esta etapa, las fresas femorales adecuadas al implante elegido se insertan secuencialmente, cada una debe insertarse hasta el plano del corte femoral. Esta preparación se considera completa cuando la última fresa insertada alcanza el nivel preoperatorio previsto. Debe preservarse la anteversión del implante en relación al eje femoral. Una vez colocado el componente femoral de prueba, se selecciona una cabeza femoral. Su altura depende de la posición del implante con referencia a la posición preoperatoria. El uso de fluoroscopia es útil para corroborar la posición del componente dentro del canal femoral y la distancia con respecto al componente acetabular. Se realiza la reducción del componente femoral con ayuda de un asistente que manipule la mesa especial con tracción y rotación interna progresiva. Una vez lograda la reducción, la tracción debe liberarse completamente, la posición de la rodilla es una adecuada referencia y la estabilidad del sistema protésico debe ser verificada en todos los movimientos antes de colocar los implantes definitivos.

Una vez verificada la estabilidad, se realiza de nuevo rotación externa y una luxación asistida de los componentes, se posiciona de nuevo la extremidad en extensión y rotación externa entre 90 y 110 grados y aducción. Se retira el componente femoral de prueba y se coloca el definitivo, se puede colocar la cabeza definitiva o, a consideración del cirujano, realizar pruebas de nuevo para verificar longitud y estabilidad con cabezas de prueba. Una vez seleccionada la adecuada, se cambia la cabeza de prueba por la definitiva y se realiza de nuevo reducción de los implantes. Se verifica de nuevo la estabilidad de la prótesis realizando movimientos de rotación externa e interna, extensión y flexión (Figura 4).

En este paso, se puede separar la bota de la mesa y realizar movimientos libres con la rodilla a 90 grados de flexión. Se fija de nuevo la bota a la mesa. Pueden obtenerse diversas vistas con el intensificador de imágenes durante las pruebas de estabilidad, verificando además la longitud de la extremidad y la posición de los diversos componentes. 


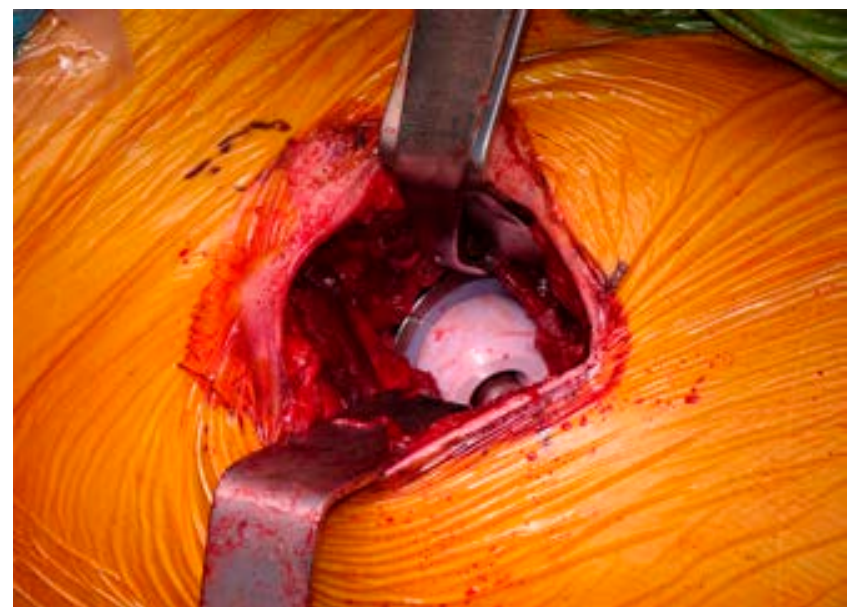

Figura 4: Prótesis colocada. Verificación de movimientos.

\section{Cierre}

El cierre va precedido de una adecuada hemostasia y lavado exhaustivo con solución. Es conveniente verificar que no exista un sangrado en la porción anterior de la cápsula posterior. Este cierre se realiza en cuatro etapas: la cápsula articular en su posición anatómica referida con dos puntos simples, es posible colocar drenaje en este plano; la fascia del tensor de la fascia lata; un plano subcutáneo; y la piel con grapas o la sutura de preferencia del cirujano. Se cubre la herida con un parche especial y se retiran los campos quirúrgicos.

Se realiza una radiografía de control antes de retirar al paciente de la mesa verificando la posición final de los implantes (Figura 5). Debe tenerse un especial cuidado al retirar al paciente de la mesa quirúrgica, ya que al retirar el poste de la región perineal y desmontar la o las botas, dependiendo de la mesa empleada, el paciente queda sin ningún tipo de sujeción y con el riesgo de tener una caída de la mesa, por lo que no debe descuidarse en ningún momento hasta colocarlo en la cama hospitalaria.

\section{Cuidados postquirúrgicos}

Los cuidados postoperatorios son similares a los realizados en cualquier tipo de artroplastia, pero con menor restricción de movimientos de rotación interna o flexión. Se inicia profilaxis antitrombótica y antibiótica. La reeducación de la marcha inicia en las primeras 12 a 24 horas del postoperatorio, de preferencia asistida por andadera de tipo mixta. Ésta se recomienda por una semana y se progresa a asistencia con bastón de un punto. Es posible ejercitar inmediatamente los músculos glúteos que no se han visto afectados por el abordaje.

La selección del abordaje quirúrgico se ha convertido en un tema de continua discusión en cirugía de cadera. El abordaje anterior ofrece diversas ventajas a corto y mediano plazo, comparado con abordaje lateral o posterior. Diversos estudios han demostrado que a largo plazo las diferencias no son estadísticamente significativas, pero la técnica de mínima invasión es conveniente para diversos grupos de pacientes en los que la rápida recuperación es un factor en la reincorporación a sus actividades y a su calidad de vida, secundario a la disminución del dolor postoperatorio, conservación del tono muscular durante el postoperatorio inmediato, disminución de la pérdida de sangre, hospitalización más breve y un riesgo reducido de luxación, así como un menor costo total. ${ }^{9}$

$\mathrm{El}$ abordaje anterior se puede utilizar para casos con casi todo tipo de morfología corporal y afecciones de cadera. Algunas características anatómicas de la cadera y la pelvis nativas son reconocidas para hacer un abordaje anterior más difícil, por ejemplo, la protrusión acetabular acerca al canal femoral hacia la pelvis y puede limitar el acceso al fémur, un ángulo cervicodiafisario con un offset disminuido posiciona el canal femoral más profundo en el muslo; factores asociados con la musculatura de pacientes obesos pueden limitar la exposición. ${ }^{10-13}$

Una desventaja del abordaje anterior es el acceso limitado a la columna posterior. Si un paciente presenta material de osteosíntesis en la columna posterior, o existe pérdida ósea posterior que requiriese

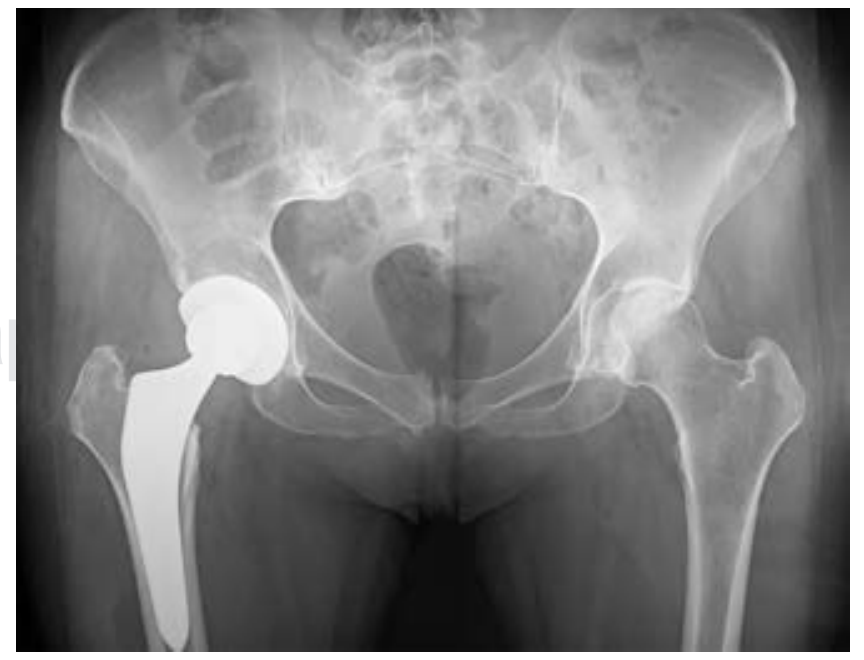

Figura 5: Radiografía anteroposterior de pelvis. Artroplastia total de cadera derecha con sistema no cementado AMIS (Medacta internacional). 
aumento, la exposición lograda por el abordaje anterior puede resultar inadecuada. ${ }^{13-17}$

El abordaje presentado en esta revisión tiene diferencias significativas en relación con el abordaje anterior directo, el cual se realiza entre el recto anterior y el músculo sartorio. La técnica se ha simplificado haciendo énfasis en una disección cuidadosa del plano intermuscular e identificación de la cápsula articular. No se requiere el uso de separadores excesivos con lo que disminuye el riesgo a lesión de tejidos blandos, la osteotomía de la cabeza femoral se realiza in situ con un solo corte y sin luxarla previamente. El uso de una mesa especial facilita la movilización de la extremidad y el posicionamiento del fémur durante el procedimiento y en la realización de pruebas de estabilidad de la prótesis

La técnica es fácilmente reproducible y puede ser realizada por el cirujano y un solo ayudante.

Como en cualquier procedimiento quirúrgico, se requiere de una curva de aprendizaje asociada con la posibilidad de complicaciones durante los primeros casos, ${ }^{18,19}$ por lo cual se recomienda la asesoría de un cirujano experimentado en la técnica durante las etapas de transición de un cirujano acostumbrado a otro abordaje. De igual forma, la incorporación de esta técnica en la formación de las nuevas generaciones de residentes favorecerá para que cirujanos más jóvenes incorporen el abordaje anterior directo modificado a su práctica cotidiana.

\section{CONCLUSIÓN}

El abordaje anterior directo modificado en artroplastia de cadera presenta diversas ventajas a corto y mediano plazo comparado con otros. Es seguro y eficaz en el manejo de la mayor parte de las patologías de la cadera como la artrosis, necrosis avascular y fracturas intracapsulares del extremoproximal del fémur, y puede emplearse con seguridad en casos de complexiones diferentes. Puede significar un mayor costo por incremento en el tiempo quirúrgico durante los primeros casos, pero en general el costo total de hospitalización disminuye al requerir de una menor estancia hospitalaria, así como los requerimientos de analgesia y terapia física.

Los resultados hacen de este abordaje una buena opción para el cirujano de cadera. Esto nos llevará a continuar difundiendo la técnica y entrenando más cirujanos, junto con otros factores, para impulsar mejores resultados para los pacientes que requieren una artroplastia de cadera.

\section{REFERENCIAS}

1. Rachbauer F, Kain MS, Leunig M. The history of the anterior approach to the hip. Orthop Clin North Am. 2009; 40 (3): 311-320.

2. Light TR, Keggi KJ. Anterior approach to hip arthroplasty. Clin Orthop Relat Res. 1980; (152): 255-260.

3. Post ZD, Orozco F, Diaz-Ledezma C, Hozack WJ, Ong A. Direct anterior approach for total hip arthroplasty: indications, technique, and results. J Am Acad Orthop Surg. 2014; 22 (9): 595-603.

4. Mast NH, Laude F. Revision total hip arthroplasty performed through the Hueter interval. J Bone Joint Surg Am. 2011; 93 Suppl 2: 143-148.

5. Matta JM, Ferguson TA. The anterior approach for hip replacement. Orthopedics. 2005; 28 (9): 927-928.

6. Matta JM, Shahrdar C, Ferguson T. Single-incision anterior approach for total hip arthroplasty on an orthopaedic table. Clin Orthop Relat Res. 2005; 441: 115-124.

7. Laude F, Candiotto S, Faldini C. AMIS publication review. M.O.R.E. Institute, Medacta International Journal. 2016; Suppl 1.

8. Diego-Ball D, Cabal AA. Descripción anatómica del abordaje anterior de cadera: seguridad y efecto para artroplastía total. Acta Ortop Mex. 2020; 34 (2): 123-128.

9. Petis SM, Howard JL, Lanting BA, Marsh JD, Vasarhelyi EM. In-hospital cost analysis of total hip arthroplasty: does surgical approach matter? J Arthroplasty. 2016; 31 (1): 53-58. doi: 10.1016/j.arth.2015.08.034.

10. Sebecic B, Staresinic M, Culjak V, Japjec M. Minimally invasive hip arthroplasty: advantages and disadvantages. Med Glas (Zenica). 2012; 9 (1): 160-165.

11. Cadossi M, Sambri A, Tedesco G, Mazzotti A, Terrando S, Faldini C. Anterior approach in total hip replacement. Orthopedics. 2017; 40 (3): e553-e556.

12. Skowronek P, Wojciechowski A, Wypniewski K, Sibinski M, Polguj M, Maksymiuk-Klos A et al. Time efficiency of direct anterior hip arthroplasty compared to postero-lateral approach in elderly patients. Arch Med Sci. 2021; 17 (1): 106-112.

13. Kyriakopoulos G, Poultsides L, Christofilopoulos P. Total hip arthroplasty through an anterior approach: The pros and cons. EFORT Open Rev. 2018; 3 (11): 574-583.

14. Free MD, Owen DH, Agius PA, Pascoe EM, Harvie P. Direct anterior approach total hip arthroplasty: an adjunct to an enhanced recovery pathway: outcomes and learning curve effects in surgeons transitioning from other surgical approaches. J Arthroplasty. 2018; 33 (11): 3490-3495.

15. Patel NN, Shah JA, Erens GA. Current trends in clinical practice for the direct anterior approach total hip arthroplasty. J Arthroplasty. 2019; 34 (9): 1987-1993.e3.

16. Wang Z, Bao HW, Hou JZ. Direct anterior versus lateral approaches for clinical outcomes after total hip arthroplasty: a meta-analysis. J Orthop Surg Res. 2019; 14 (1): 63.

17. Haller JM, Working ZM, Ross HL, Gililland JM, Kubiak EN. Fewer hip dislocations with anterior total hip arthroplasty for displaced femoral neck fracture. Orthopedics. 2021; 44 (2): e248-e252.

18. de Steiger RN, Lorimer M, Solomon M. What is the learning curve for the anterior approach for total hip arthroplasty? Clin Orthop Relat Res. 2015; 473 (12): 3860-3866.

19. Brun OL, Mansson L, Nordsletten L. The direct anterior minimal invasive approach in total hip replacement: a prospective departmental study on the learning curve. Hip Int. 2018; 28 (2): 156-160. 